\title{
Identification of Ebola virus microRNAs and their putative pathological function
}

\author{
LIANG HongWei ${ }^{\dagger}$, ZHOU Zhen ${ }^{\dagger}$, ZHANG SuYang ${ }^{\dagger}$, ZEN Ke $^{*}$, CHEN Xi ${ }^{*}$ \\ \& ZHANG ChenYu*
}

Jiangsu Engineering Research Center for microRNA Biology and Biotechnology, State Key Laboratory of Pharmaceutical Biotechnology, School of Life Sciences, Nanjing University, Nanjing 210093, China

Received September 5, 2014; accepted September 13, 2014; published online September 28, 2014

\begin{abstract}
Ebola virus (EBOV), a member of the filovirus family, is an enveloped negative-sense RNA virus that causes lethal infections in humans and primates. Recently, more than 1000 people have been killed by the Ebola virus disease in Africa, yet no specific treatment or diagnostic tests for EBOV are available. In this study, we identified two putative viral microRNA precursors (pre-miRNAs) and three putative mature microRNAs (miRNAs) derived from the EBOV genome. The production of the EBOV miRNAs was further validated in HEK293T cells transfected with a pcDNA6.2-GW/EmGFP-EBOV-pre-miRNA plasmid, indicating that EBOV miRNAs can be produced through the cellular miRNA processing machinery. We also predicted the potential target genes of these EBOV miRNAs and their possible biological functions. Overall, this study reports for the first time that EBOV may produce miRNAs, which could serve as non-invasive biomarkers for the diagnosis and prognosis of EBOV infection and as therapeutic targets for Ebola viral infection treatment.
\end{abstract}

microRNA, Ebola virus, function

Citation: $\quad$ Liang HW, Zhou Z, Zhang SY, Zen K, Chen X, Zhang CY. Identification of Ebola virus microRNAs and their putative pathological function. Sci China Life Sci, 2014, 57: 973-981, doi: 10.1007/s11427-014-4759-2

The Ebola virus (EBOV), a member of the filovirus family, is an enveloped negative-sense RNA virus that causes severe and frequently lethal infections in humans and primates [1]. Recently, more than 1000 people have been killed by Ebola virus disease in Africa, and many other areas (e.g., Benin, America, and Canada) have also reported suspected cases [2,3]. The disease has a high risk of death, killing between $50 \%$ and $90 \%$ of those infected with the virus [4]. However, no specific treatment or diagnostic tests for EBOV are currently available [5].

As a class of small non-coding RNAs ranging from 19 to 24 nucleotides (nt) in length, microRNAs (miRNAs) regu-

$\dagger$ Contributed equally to this work

*Corresponding author (email: cyzhang@nju.edu.cn; xichen@nju.edu.cn; kzen@nju.edu.cn) late the expression of target genes at the posttranscriptional level [6-8]. miRNAs are not only found in numerous eukaryotes but are also employed by viruses to regulate the expression of their own genes, the host's genes, or both [9-11]. Most viral miRNAs have been identified from virus-infected cells using traditional cloning strategies [12-15], although some have been identified following computational prediction and hybridization analysis [15-17]. Currently, the miRBase database lists 30,424 miRNAs [18,19], of which 501 are viral miRNAs. It is worth noting that the majority of known viral miRNAs are encoded by DNA viruses. Indeed, DNA-virus-encoded miRNAs are generally accepted, as viruses with DNA genomes replicate in the nucleus and thus have access to the cellular miRNA processing machinery; in contrast, RNA 
viruses replicate in the cytoplasm and, therefore, are not expected to encode miRNAs [9-11]. It has also been speculated that RNA viruses do not generate miRNAs to avoid the adverse effects caused by the miRNA processing machinery [11]. Therefore, naturally occurring miRNAs derived from RNA viruses have not been widely acknowledged. Despite the theoretical barriers preventing RNA viruses from encoding miRNAs, recent studies have confirmed that laboratory-engineered RNA viruses, including the influenza virus, sindbis virus, and vesicular stomatitis virus (VSV), are capable of expressing miRNA-like small RNAs [20-25]. Furthermore, a few retroviruses, including the human immunodeficiency virus (HIV) [26,27], bovine leukemia virus (BLV) $[28,29]$ and three cytoplasmic RNA viruses, the West Nile virus (WNV) [30], Dengue virus (DENV) [31] and hepatitis A virus (HAV) [20], were found to encode miRNAs, although the underlying mechanisms remain unclear.

In this study, we hypothesized that the genome of Ebola virus could produce miRNAs using the cellular miRNA processing machinery. To validate this, we used a strategy that combined computational prediction and experimental validation to search for putative EBOV miRNAs. This study provides evidence to support the hypothesis that EBOV can encode functional miRNAs through the cellular miRNA processing machinery. In addition, our findings provide a basis for further assessing the roles of the EBOV miRNAs during viral infection and virus-host interactions.

\section{Materials and methods}

\subsection{Cells, plasmids and reagents}

HEK293T cells were purchased from Thermo Scientific (Cat No. HCL4517) and grown in Dulbecco's modified Eagle's medium (DMEM) (high glucose formulation, Gibco Life Technologies, Grand Island, NY, USA) supplemented with 10\% fetal calf serum (Gibco Life Technologies, Grand Island, NY, USA) at $37^{\circ} \mathrm{C}$ in a humid environment containing $5 \% \mathrm{CO}_{2}$. The eukaryotic expression plasmids, pcDNA6.2-GW/EmGFP-EBOV-pre-miR-1 and pcDNA6.2GW/EmGFP-EBOV-pre-miR-2, and the wild-type and mutant sensor plasmids (pmirGLO-EBOV-miRNA-wild and pmirGLO-EBOV-miRNA-mut) were purchased from GenePharma (Shanghai, China). All constructs generated were confirmed by sequencing using universal primers (BGI, Guangzhou, China). The mouse monoclonal anti-Dicer1 antibody 5D12.2 (1:5000 dilution; mouse monoclonal; Millipore Corporation, Billerica, MA, USA), a rabbit anti-GAPDH polyclonal antibody (G-9) (sc-365062; 1:2000 dilution; rabbit polyclonal; Santa Cruz, CA, USA), and the appropriate HRP-conjugated anti-mouse and anti-rabbit secondary antibodies (1:10,000 dilution; Proteintech Group, Inc. Chicago, IL, USA) were used for immunoblotting.

\subsection{Bioinformatics prediction of the miRNAs}

A flowchart describing the computational prediction of the putative miRNAs is shown in Figure 1. Briefly, the viral genome was scanned for stem-loop structures of miRNA precursor (pre-miRNA) using VMir [32], a computational analyzer program for the prediction of putative pre-miRNAs. The complete genome sequences of different EBOV strains, the Sudan ebolavirus strain Gulu and the Zaire ebolavirus strain Mayinga-Volchkov (GenBank accession No. NC_006432 and NC_002549), were used. VMir predictions were carried out using the default parameters. The putative pre-miRNAs that satisfied the filter parameters of a VMir score $\geqslant 150$ and a window count $\geqslant 35$ were selected for further assessment. Then, the source code of MiPred [33] was used to distinguish real and pseudo miRNA precursors from the obtained sequences with a prediction confidence equal to or greater than $70 \%$. Subsequently, mature miRNA sequences were predicted from the pre-miRNA stem-loops. To extend the prediction coverage of the mature miRNAs, we used two strategies: the MatureBayes tool [34] and the Bayes-SVM-MiRNA web server v1.0 [35]. The default conditions were used for the MatureBayes tool. The folding energy was set at $-15 \mathrm{kcal} \mathrm{mol}^{-1}$ when using the Bayes-SVM-MiRNA web server v1.0; other filter parameters were set to the default values.

\subsection{RNA interference (RNAi) of the Dicer gene}

The following siRNA sequence targeting the human Dicer cDNA was designed and synthesized by GenePharma (Shanghai, China): 5'-UAAAGUAGCUGGAAUGAUG-3'. A scrambled siRNA was included as a negative control. The Dicer siRNA was transfected into HEK293T cells using

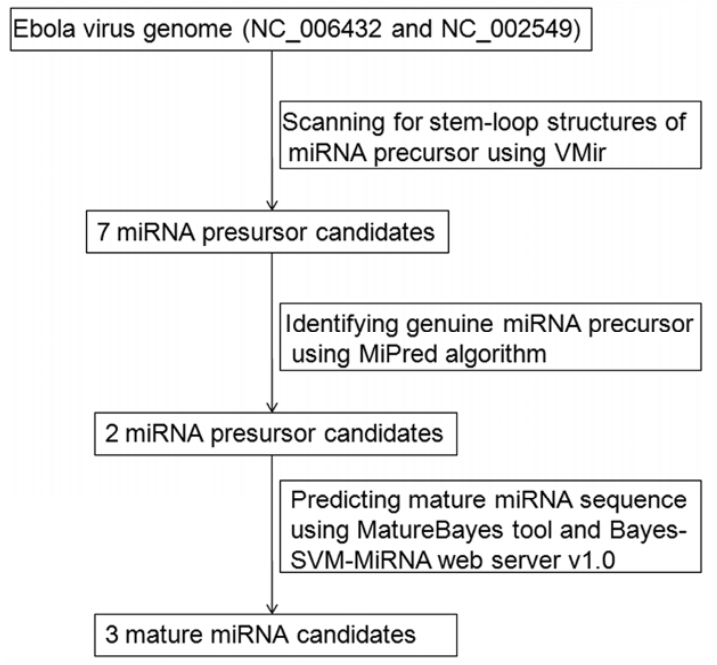

Figure 1 Flowchart of EBOV-encoded miRNA prediction. The VMir analyzer program was used to predict the putative EBOV genome-encoded pre-miRNA stem-loop structures, MiPred algorithm was used to identify genuine pre-miRNAs, and the MatureBayes tool and the Bayes-SVMMiRNA web server v1.0 were used to predict the mature miRNA sequences. 
Lipofectamine 2000 (Invitrogen, Carlsbad, CA, USA) according to the manufacturer's instructions. Total RNA and protein were isolated $24 \mathrm{~h}$ post transfection. The Dicer mRNA and protein expression levels were assessed by quantitative RT-PCR and Western blotting.

\subsection{RNA isolation and quantitative RT-PCR}

Total RNA was extracted from the cultured cells and human tissues using the TRIzol Reagent (Invitrogen) according to the manufacturer's instructions. Assays to quantify the viral miRNAs were performed using miRNA probes (GenePharma, Shanghai, China) according to the manufacturer's instructions. Briefly, $1 \mu \mathrm{g}$ of total RNA was reversetranscribed to cDNA using the AMV reverse transcriptase (TaKaRa, Dalian, China) and a stem-loop RT primer (GenePharma). The reaction conditions were as follows: $16^{\circ} \mathrm{C}$ for $30 \mathrm{~min}, 42^{\circ} \mathrm{C}$ for $30 \mathrm{~min}$, and $85^{\circ} \mathrm{C}$ for $5 \mathrm{~min}$. Real-time PCR was performed using a GenePharma PCR kit on an Applied Biosystems 7900 Sequence Detection System (Applied Biosystems). The reactions were incubated in a 96-well optical plate at $95^{\circ} \mathrm{C}$ for $10 \mathrm{~min}$, followed by 40 cycles of $95^{\circ} \mathrm{C}$ for $15 \mathrm{~s}$ and $60^{\circ} \mathrm{C}$ for $1 \mathrm{~min}$. All of the reactions were run in triplicate. After the reaction, the cycle threshold $\left(C_{\mathrm{T}}\right)$ data were determined using fixed threshold settings, and the mean $C_{\mathrm{T}}$ of the triplicate PCRs was determined. A comparative $C_{\mathrm{T}}$ method was used to compare each condition to the controls. The relative levels of the miRNAs in the cells and tissues were normalized to U6. The amount of miRNA relative to the internal control U6 was calculated using the $2^{-\Delta \Delta C_{\mathrm{T}}}$ equation, in which $\Delta \Delta C_{\mathrm{T}}=\left(C_{\mathrm{T} \text { miRNA }}{ }^{-}\right.$ $\left.C_{\mathrm{T} \mathrm{U}}\right)_{\text {target }}-\left(C_{\mathrm{T} \text { miRNA }}-C_{\mathrm{T} \mathrm{U}}\right)_{\text {control }}$.

To quantify the Dicer mRNA, $1 \mu \mathrm{g}$ of total RNA was reverse-transcribed to cDNA using oligo dT and Thermoscript (TaKaRa); reaction conditions were as follows: $42^{\circ} \mathrm{C}$ for 60 min and $70^{\circ} \mathrm{C}$ for $10 \mathrm{~min}$. Next, real-time PCR was performed using the RT product, SYBER Green Dye (Invitrogen), and specific primers for Dicer and GAPDH. The sequences of the primers were as follows: Dicer (sense): 5'TGCTATGTCGCCTTGAATGTT-3'; Dicer (antisense): 5'-AATTTCTCGATAGGGGTGGTCTA-3'; GAPDH (sense): 5'-GATATTGTTGCCATCAATGAC-3'; and GAPDH (antisense): 5'-TTGATTTTGGAGGGATCTCG-3'. The reactions were incubated at $95^{\circ} \mathrm{C}$ for $5 \mathrm{~min}$, followed by 40 cycles of $95^{\circ} \mathrm{C}$ for $30 \mathrm{~s}, 60^{\circ} \mathrm{C}$ for $30 \mathrm{~s}$, and $72^{\circ} \mathrm{C}$ for $30 \mathrm{~s}$. After the reactions were complete, the $C_{\mathrm{T}}$ values were determined by setting a fixed threshold. The relative amount of Dicer mRNA was normalized to GAPDH.

\subsection{Western blot analysis}

The total protein in the cell extracts was quantified using the BCA protein assay Kit (Pierce, Rockford, IL, USA) according to manufacturer's instructions. Thirty micrograms of total protein were resolved on $8 \%$ SDS-PAGE gels and transferred onto PVDF membranes (Millipore, Billerica, MA, USA), followed by blocking with 5\% non-fat milk at room temperature for $2 \mathrm{~h}$. Membranes were probed with the specific primary antibodies overnight at $4^{\circ} \mathrm{C}$, followed by incubation with the appropriate (anti-mouse or anti-rabbit) HRP-conjugated secondary antibodies at room temperature for $1 \mathrm{~h}$. Protein signals were visualized by ECL chemiluminescence using the Immobilon Western HRP Substrate (Millipore Corporation, Billerica, MA, USA) according to the manufacturer's protocol.

\subsection{Luciferase reporter assay}

A dual luciferase reporter assay using the sensor plasmids (pmirGLO-EBOV-miRNA-wild and pmirGLO-EBOVmiRNA-mut) was performed in HEK293T cells. Briefly, HEK293T cells were seeded at approximately $1 \times 10^{6}$ cells per well in a 24-well plate one day prior to transfection. The next day, $200 \mathrm{ng}$ of either the pmirGLO-EBOV-miRNAwild or the pmirGLO-EBOV-miRNA-mut plasmid were co-transfected with 20 pmol of chemically synthesized miRNA mimics (GenePharma, Shanghai, China) or $200 \mathrm{ng}$ of pcDNA6.2-GW/EmGFP-EBOV-pre-miRNA plasmid into the HEK293T cells with Lipofectamine 2000 (Invitrogen). The firefly and Renilla luciferase activities were evaluated simultaneously $48 \mathrm{~h}$ post-transfection using the Dual-Glo ${ }^{\mathrm{TM}}$ Luciferase Assay System (Promega, Madison, WI, USA) according to the manufacturer's protocol. Relative luciferase activity was expressed as the ratio of firefly to Renilla luciferase activity. The transfections were performed independently in triplicate.

\subsection{Statistical analysis}

Data were presented as mean \pm SE. Values from three independent experiments were analyzed by two-tailed Student's $t$-tests. $P<0.05$ was considered to be statistically significant.

\section{Results}

\subsection{Prediction of pre-miRNAs and mature miRNAs encoded by the EBOV genome}

Computational prediction represents an effective strategy to identify novel miRNAs, which can be further validated by experimental approaches. We followed this strategy and investigated whether the EBOV genome contained sequences that can fold into the stem-loop structures of pre-miRNA. Two strains of EBOV, Sudan ebolavirus (Sudan ebolavirus strain Gulu, GenBank accession No. NC_006432) and Zaire ebolavirus (Zaire ebolavirus strain Mayinga-Volchkov, GenBank accession No. NC_002549), were analyzed using the VMir analyzer program [32], and seven sequences with potential stem-loop structures were found (Figure 1). Then, the MiPred algorithm was used to 
narrow down the seven candidates using characteristics of genuine pre-miRNAs. Two putative pre-miRNAs, named EBOV-pre-miR-1 and EBOV-pre-miR-2, were retained (Table 1). The VMir analyzer program further showed that the two selected pre-miRNAs were conserved among different EBOV strains (GenBank accession No. NC_006432, AF499101, AF272001, AY142960, NC_002549, AY354458, EU224440, and EU338380), with VMir scores $\geqslant 200$ and window counts $\geqslant 50$ (Figure $2 A$ ). The RNAFold algorithm was then used to show that EBOV-pre-miR-1 and EBOV-pre-miR-2 had the typical stem-loop structure of pre-miRNAs (Figure 2B). The mature miRNAs produced by the two pre-miRNAs were predicted using the MatureBayes tool [34] and the Bayes-SVM-MiRNA web server v1.0 [35]. Both algorithms identified three putative mature miRNAs that were $22 \mathrm{nt}$ in length, namely EBOV-miR-1-5p, EBOV-miR-1-3p and EBOV-miR-2-3p (Table 2). Overall, we identified two putative pre-miRNAs and three putative mature miRNAs from the genome of EBOV using computational approaches.

\subsection{Detection of EBOV miRNAs in HEK293T cells transfected with the pcDNA6.2-GW/EmGFP-EBOV- pre-miRNA plasmid}

Next, we employed a plasmid-derived miRNA expression system to determine whether the EBOV miRNA can be processed by the cellular miRNA processing machinery. EBOV-pre-miR-1 and EBOV-pre-miR-2 were cloned into the mammalian expression plasmid pcDNA6.2-GW/ EmGFP to generate the recombinant pcDNA6.2-GW/ EmGFP-EBOV-pre-miRNA plasmid, which was then transfected into HEK293T cells (Figure 3A). A plasmid that expressed scrambled small RNA was also transfected into HEK293T cells as the negative control. A strong fluorescent signal was observed under fluorescence microscopy in both transfected cell groups (Figure 3B). RT-PCR analysis re- vealed that the EBOV miRNAs were expressed as an apparent band of $100 \mathrm{bp}$ in HEK293T cells transfected with the pcDNA6.2-GW/EmGFP-EBOV-pre-miRNA (Figure 3C). In contrast, no bands were detected in cells transfected with the negative control plasmid. Sequence analysis of the PCR product indicated that the PCR-amplified sequence was identical to the EBOV miRNAs.

To determine whether the EBOV miRNAs were generated using the cellular miRNA processing pathway, we silenced Dicer using an RNAi approach in HEK293T cells. Efficient knockdown of Dicer was confirmed by qRT-PCR and Western blotting (Supplementary Figure S1). Dicerdeficient and wild-type HEK293T cells were transfected with the pcDNA6.2-GW/EmGFP-EBOV-pre-miRNA plasmid, and qRT-PCR analysis was performed to measure the levels of the EBOV miRNAs. A dramatic reduction in the EBOV miRNA levels was found in Dicer-deficient cells compared with wild-type cells (Figure 3D), suggesting that the generation of EBOV miRNA is dependent on the Dicer processing pathway.

\subsection{EBOV-encoded miRNAs are biologically functional}

miRNAs usually negatively regulate gene expression by binding to the $3^{\prime}$-untranslated region (3'-UTR) of target mRNA molecules $[30,36]$. To determine whether the EBOV miRNAs are functionally active, we predicted the target genes of EBOV miRNAs using Targetscan [37] and RNAhydrid [38] (Supplementary Table S1). According to GO analysis, the EBOV miRNAs have the ability to inhibit human genes involved in $\mathrm{T}$ and $\mathrm{B}$ cell activation, nature killer cell-mediated cytotoxicity, adherens junction, and apoptosis. These results strongly imply that immune escape, endothelial cell rupture and tissue dissolution during EBOV infection is due to the action of the EBOV miRNAs.

Subsequently, a dual-luciferase reporter assay using either the pmirGLO-EBOV-miRNA-wild or the pmirGLOEBOV-miRNA-mut plasmid was performed in HEK293T

Table 1 The predicted pre-miRNAs in EBOV

\begin{tabular}{ccccc}
\hline Pre-miRNA & Sequence (5'-3') & Strand & $\begin{array}{c}\text { Start } \\
\text { position }\end{array}$ & $\begin{array}{c}\text { Length } \\
\text { Minimum of free } \\
\text { energy }\left(\mathrm{kcal}_{\text {mol }}{ }^{-1}\right)\end{array}$ \\
\hline $\begin{array}{c}\text { EBOV- } \\
\text { pre-miR-1 }\end{array}$ & $\begin{array}{c}\text { AUAGAACGAGGAAGAUUAAGAAAAAGUCCAUAAUGCUGGGG } \\
\text { AGGCAAUCCUUGCCACCAUAGGACUUUUUCAAUUCCUCUAUU } \\
\text { UUAU }\end{array}$ & + & 11450 & 87 \\
\hline $\begin{array}{c}\text { EBOV- } \\
\text { pre-miR-2 }\end{array}$ & $\begin{array}{c}\text { AUAAACCUCUUGAUUUCGGGACCAUUGCACUAUCCUUAGCAG } \\
\text { UUCCUCAGGUAUUGGGUGGAUUAUCCUUCCUAAAUCCAGAAA } \\
\text { AGUGC }\end{array}$ & + & 14252 & 89 \\
\hline
\end{tabular}

Table 2 The predicted mature miRNAs in EBOV

\begin{tabular}{llc}
\hline Mature miRNA & Sequence $\left(5^{\prime}-3^{\prime}\right)$ & Length \\
\hline EBV-miR-1-5p & AAAAAGUCCAUAAUGCUGGGGA & 22 \\
EBV-miR-1-3p & GCCACCAUAGGACUUUUUCAAU & 22 \\
EBV-miR-2-3p & UUAUCCUUCUUGAAUCCUGAGA & 22 \\
\hline
\end{tabular}



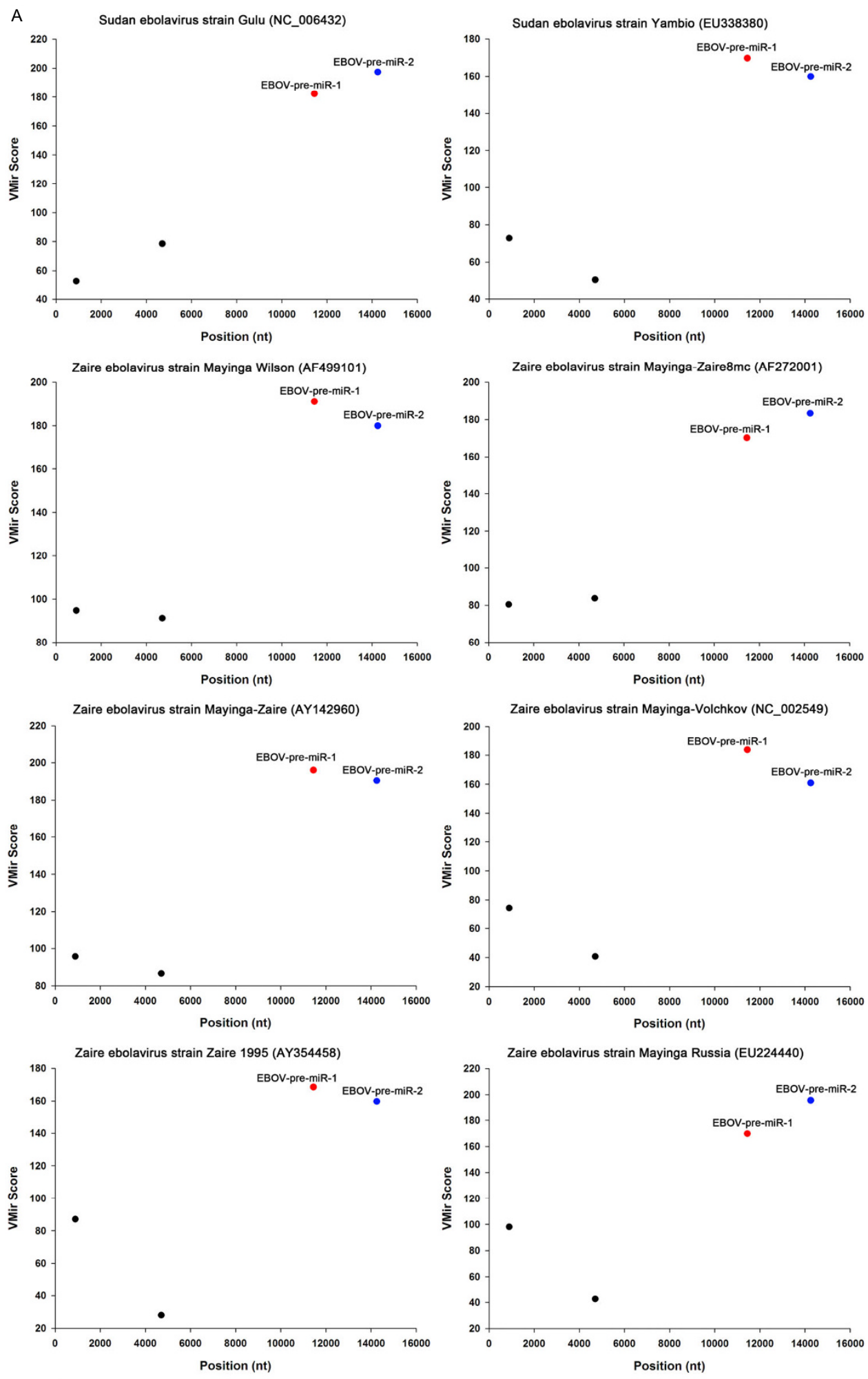

B EBOV-pre-miR-1

EBOV-pre-miR-2

$$
\begin{aligned}
& \text { 5'--AU AC GAUUAA AUA C G C } \\
& \text { AGA GAGGAA GAAAAAGUCC AUG UGG GAGG A } \\
& \text { 3'-UAU AUU CUCCUU CUUUUUCAGG UAC ACC UUCC A }
\end{aligned}
$$

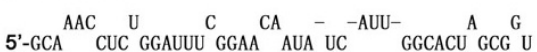$$
\begin{array}{llll}
\text { 5'-GCA } & \text { CUC GGAUUU GGAA } & \text { AUA UC } & \text { GGCACU GCG U } \\
\text { 3'-UGU } & \text { GAG CCUAAG UCUU } & \text { UAU GG } & \text { UCGUGG CGC }
\end{array}
$$

Figure 2 VMir prediction of EBOV-encoded pre-miRNAs. A, The two candidate pre-miRNAs with high scores are colored red (EBOV-pre-miR-1) and blue (EBOV-pre-miR-2). The VMir analyzer program was used to predict candidate pre-miRNAs in the EBOV genome with a cutoff score of 150 and a cutoff window count of 35 . The $y$-axis represents the VMir scores, while the x-axis indicates the nucleotide position. B, Secondary structures of the predicted candidate EBOV pre-miRNAs. Mature miRNAs in the $5^{\prime}$ and $3^{\prime}$ strands are shown in red and blue, respectively. 


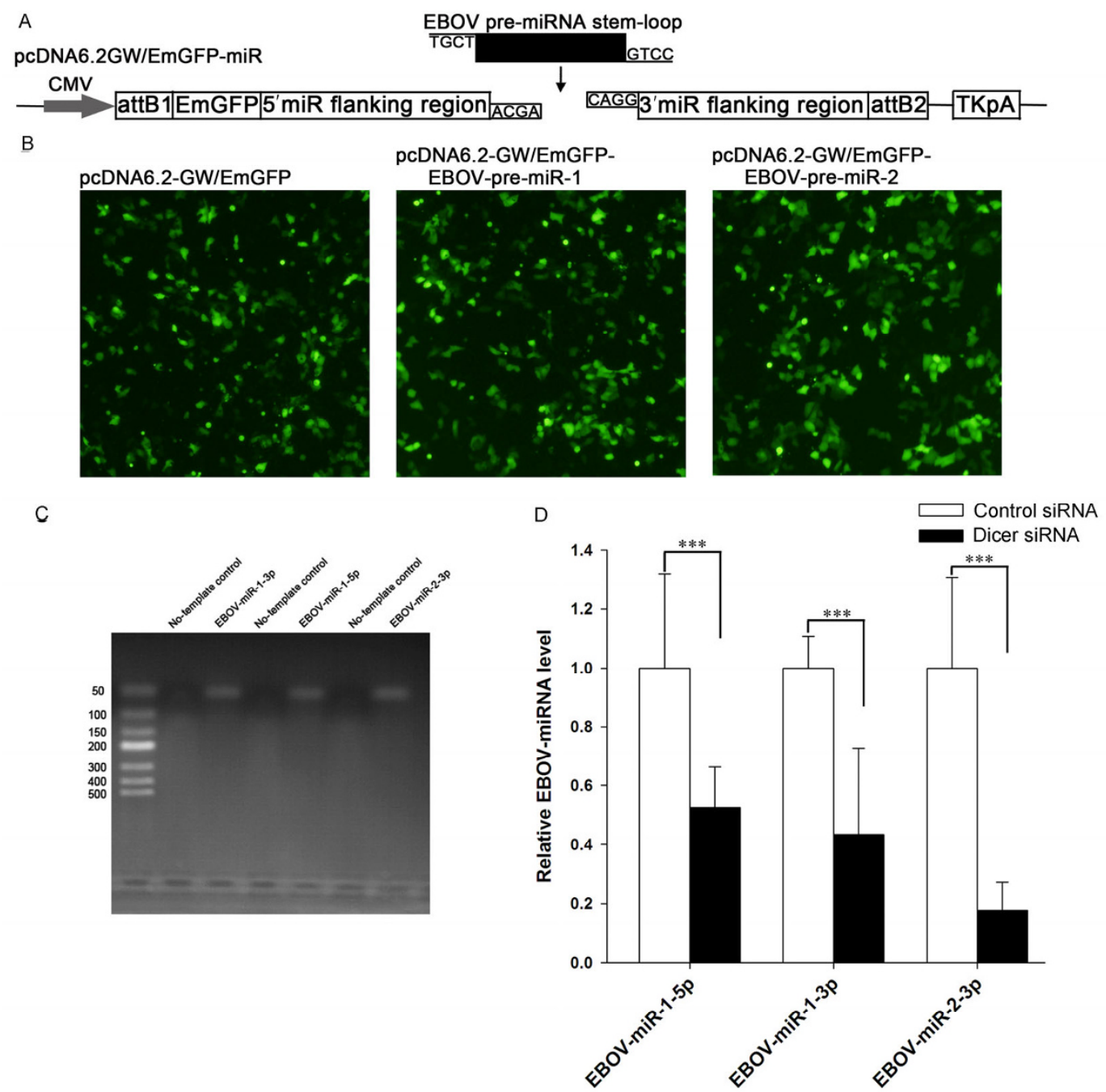

Figure 3 Detection of EBOV miRNAs from plasmid-expressed precursor miRNA (pre-miRNA). A, Construction of the EBOV miRNA expression plasmid. Each EBOV pre-miRNA sequence was cloned into the pcDNA6.2-GW/EmGFP plasmid. A plasmid containing scrambled sequences was used as a negative control. B, HEK293T cells were transfected with the miRNA expression plasmids, and strong green fluorescence was observed in both transfected cell lines $(100 \times)$. C, Detection of mature miRNA expression. Small RNAs were harvested at $24 \mathrm{~h}$ post-transfection, and mature miRNAs were detected by stem-loop RT-PCR in both transfected cell lines. D, Plasmid-expressed miRNA levels were reduced in Dicer-deficient HEK293T cells compared to wild-type negative control cells. Significant differences in the expression levels were determined by Student's $t$-test $(*, P<0.05 ; * *, P<0.01 ; * * *, P<0.001)$.

cells (Figure 4A). First, we tested the effect of synthetic EBOV miRNA mimics on the expression of the luciferase gene containing artificial targets of EBOV miRNA. The efficient overexpression of EBOV miRNAs in HEK293T cells is shown in Supplementary Figure S2A. Luciferase activity was significantly reduced when cells were cotransfected with the EBOV miRNA mimics and the pmirGLO-EBOV-miRNA-wild plasmid compared to those co-transfected with the mimics and mutant plasmid (Figure 4B). Second, we co-transfected the pcDNA6.2-GW/ EmGFP-EBOV-pre-miRNA plasmid with the pmirGLOEBOV-miRNA-wild/mut plasmid into HEK293T cells. The efficient overexpression of the EBOV miRNAs in HEK293T cells is shown in Supplementary Figure S2B. A dramatic decrease in luciferase activity was observed in the cells transfected with the pcDNA6.2-GW/EmGFP-EBOV-
pre-miRNA plasmid (Figure 4C). These data suggest that the EBOV miRNAs are capable of silencing the expression of the luciferase gene containing artificial targets of the EBOV miRNAs. Overall, these results suggest that the EBOV-encoded miRNAs are functionally active.

\section{Discussion}

According to the criteria proposed by Ambros et al. [39], small RNAs can be defined as genuine miRNAs if they fulfill a combination of structural and expression criteria. Here, putative EBOV miRNAs were defined as bona fide miRNAs in that they fulfilled these annotation criteria. In addition, they were identified as genuine miRNAs based on the miRNA biogenesis pathway. Similar to previously identi- 
A

\begin{tabular}{|c|c|c|c|}
\hline \multirow{2}{*}{ PGK } & \multicolumn{3}{|c|}{ pmirGLO-Fluc/hRluc } \\
\hline & Luc & MSC & polyA \\
\hline \multicolumn{4}{|c|}{$\widehat{\text { wild 5'-UCCCCAGCAUUAUGGACUUUUUU-3' }}$} \\
\hline \multicolumn{4}{|c|}{ EBOV-miR-1-5p 3'-AGGGGUCGUAAUACCUGAAAAA-5' } \\
\hline \multicolumn{4}{|c|}{ mut 5'-UCCCCAGCAUUAUGGCUGAAAAU-3' } \\
\hline \multicolumn{4}{|c|}{ wild 5'-AUUGAAAAAGUCCUAUd sequence- } \\
\hline \multirow{2}{*}{\multicolumn{4}{|c|}{$\begin{array}{r}\text { EBOV-miR-1-3p 3'-UAACUUUUUUCAGGAUACCACCG-5' } \\
\text { mut 5'-AUUGAAAAAGUCCUUACCACCC-3' }\end{array}$}} \\
\hline & & & \\
\hline \multicolumn{4}{|c|}{$\begin{array}{l}\text { sed sequence } \\
\text { wild 5'-UCUCAGGAUUCAAGAAGGAUA-3' }\end{array}$} \\
\hline \multicolumn{4}{|c|}{ EBOV-miR-2-3p 3'-AGAGUCCUAAGUUCUUCCUAUU-5' } \\
\hline & & & \\
\hline
\end{tabular}

B
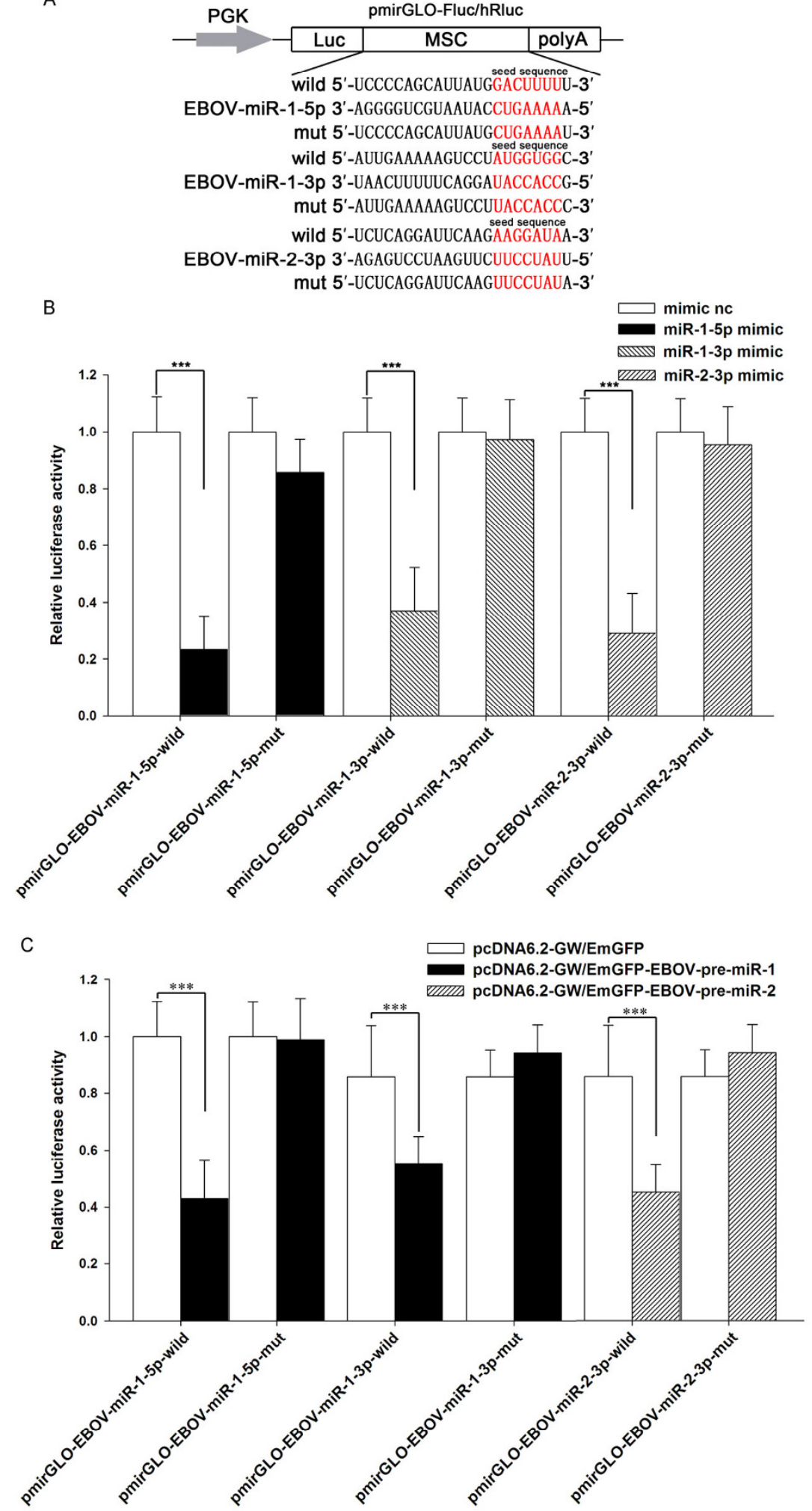

Figure 4 EBOV-encoded miRNAs are biologically functional. A, Construction of the wild and mut plasmids. The wild-type construct was created by inserting two tandem repeats of artificial target sites that were perfectly complementary to the miRNA sequences into the $3^{\prime}$ UTR of the luciferase gene in the sensor plasmid, and the mutant construct was created by inserting two tandem repeats containing mutated nucleotides in the artificial target sites. B, Silencing activity of the exogenous viral miRNAs in HEK293T cells transfected with viral miRNA mimics. C, Silencing activity of the EBOV miRNAs in cells transfected with the sensor plasmid and with the pcDNA6.2-GW/EmGFP-EBOV-pre-miRNA plasmid. Luciferase activity was assessed $48 \mathrm{~h}$ after transfection. For all treatments, relative luciferase activity was expressed as ratio of firefly to Renilla luciferase activity. Error bars indicate the standard deviation obtained from three replicates, and significant differences are indicated with * for $P<0.05$, ** for $P<0.01$ and *** for $P<0.001$. 
fied viral miRNAs from WNV [24], DENV [31], and HAV [20], silencing Dicer led to a significant decline in the EBOV miRNA levels. These findings suggest that a cytoplasmic RNA virus can utilize the cellular miRNA processing machinery to produce its own miRNAs.

Although several novel miRNAs have been experimentally identified in the WNV [30], DENV [31] and HAV [20] genomes, it remains controversial whether a virus with an RNA genome that replicates in the cytoplasm can naturally encode functional miRNAs or miRNA-like small RNAs. Computational prediction followed by experimental validation constitutes an effective and fast strategy for the discovery of novel miRNAs, and several studies have previously employed this strategy to identify viral miRNAs in numerous viruses [20,40-42]. In this study, two miRNA precursors, EBOV-pre-miR-1 and EBOV-pre-miR-2, were identified using the same strategy and were shown to be completely conserved among different EBOV strains. The findings provide new evidence that cytoplasmic RNA viruses can encode functional miRNAs.

Until now, there has been no specific diagnostic test for EBOV. Recently, we and other groups have demonstrated that miRNAs circulate in a highly stable, cell-free form in body fluids and that circulating miRNAs can be used as non-invasive biomarkers for molecular diagnostics and prognostics. The identification of EBOV miRNAs suggests opportunities for the development of circulating EBOV miRNAs as non-invasive biomarkers for the molecular diagnosis of EBOV infection at an early stage.

Further investigations will be needed to determine the mechanisms by which the EBOV genome encodes miRNA-like small RNAs and the potential biological function of the viral miRNAs during infection and host-virus interactions. Nevertheless, the preliminary prediction that EBOV hosts express EBOV miRNA target genes provides some clues for better understanding the regulatory roles of EBOV miRNAs. Ebola causes highly lethal hemorrhagic fever in humans, with no licensed countermeasures [43]. Its virulence can be attributed to several immunoevasion mechanisms: the early inhibition of innate immunity initiated by the downregulation of type I interferon, epitope masking, and subversion of the adaptive humoral immunity by secreting a truncated form of the viral glycoprotein [43]. Deficiencies in both specific and non-specific antiviral responses result in unrestricted viral replication and dissemination in the host, typically causing death within 10 days after the appearance of symptoms [43]. The results of cellular target prediction analysis indicate that EBOV miRNAs could regulate the expression of host genes involved in virus-cell interaction, immune escape, and cell apoptosis, which may explain how the Ebola virus can mass duplicate without detection by the host immune system and cause severe hemorrhagic fever symptoms. Interestingly, we found that the Reston EBOV strain did not encode these miRNAs, in contrast to the Sudan EBOV and Zaire EBOV strains. Unlike the Reston EBOV strain, the Sudan EBOV and Zaire EBOV strains have high pathogenicity and mortality rates in humans; the miRNAs encoded by EBOV may explain these differences, at least in part.

In summary, we have identified two putative viral pre-miRNAs and three mature miRNAs derived from EBOV and showed that the EBOV miRNAs can be generated using the cellular miRNA processing machinery. The EBOV miRNAs may have potential as non-invasive biomarkers for the molecular diagnosis and prognosis of EBOV infection and as therapeutic targets for the treatment of EBOV disease.

The authors declare they have no conflict of interest.

This work was supported by the National Basic Research Program of China (2014CB542300), the National Natural Science Foundation of China (81101330, 31271378, 81250044), the Natural Science Foundation of Jiangsu Province (BK2012014) and the Research Special Fund for Public Welfare Industry of Health (201302018). This work was also supported by the Program for New Century Excellent Talents in University from Ministry of Education of China (NCET-12-0261).

1 Feldmann H, Geisbert TW. Ebola haemorrhagic fever. Lancet, 2011, 377: 849-862

2 Frieden TR, Damon I, Bell BP, Kenyon T, Nichol S. Ebola 2014new challenges, new global response and responsibility. N Engl J Med, 2014, doi: 10.1056/NEJMp1409903

3 Dixon MG, Schafer IJ. Ebola viral disease outbreak-West Africa, 2014. MMWR Morb Mortal Wkly Rep, 2014, 63: 548-551

4 Ebola: the virus and the disease. Wkly Epidemiol Rec, 1999, 74: 89

5 Turner C. Ebola virus disease: an emerging threat. Nursing, 2014, 44: 68-69

6 Bartel DP. MicroRNAs: genomics, biogenesis, mechanism, and function. Cell, 2004, 116: 281-297

7 Ambros V. The functions of animal microRNAs. Nature, 2004, 431: 350-355

8 He L, Hannon GJ. MicroRNAs: small RNAs with a big role in gene regulation. Nat Rev Genet, 2004, 5: 522-531

9 Cullen BR. Viruses and microRNAs. Nat Genet, 2006, 38(Suppl): S25-30

10 Grundhoff A, Sullivan CS. Virus-encoded microRNAs. Virology, 2011, 411: 325-343

11 Kincaid RP, Sullivan CS. Virus-encoded microRNAs: an overview and a look to the future. PLoS Pathog, 2012, 8: e1003018

12 Pfeffer S, Zavolan M, Grasser FA, Chien M, Russo JJ, Ju J, John B, Enright AJ, Marks D, Sander C, Tuschl T. Identification of virus-encoded microRNAs. Science, 2004, 304: 734-736

13 Cai X, Lu S, Zhang Z, Gonzalez CM, Damania B, Cullen BR. Kaposi's sarcoma-associated herpesvirus expresses an array of viral microRNAs in latently infected cells. Proc Natl Acad Sci USA, 2005, 102: 5570-5575

14 Samols MA, Hu J, Skalsky RL, Renne R. Cloning and identification of a microRNA cluster within the latency-associated region of kaposi's sarcoma-associated herpesvirus. J Virol, 2005, 79: 9301-9305

15 Pfeffer S, Sewer A, Lagos-Quintana M, Sheridan R, Sander C, Grasser FA, van Dyk LF, Ho CK, Shuman S, Chien M, Russo JJ, Ju J, Randall G, Lindenbach BD, Rice CM, Simon V, Ho DD, Zavolan M, Tuschl T. Identification of microRNAs of the herpesvirus family. Nat Methods, 2005, 2: 269-276

16 Sullivan CS, Grundhoff AT, Tevethia S, Pipas JM, Ganem D. SV40-encoded microRNAs regulate viral gene expression and reduce 
susceptibility to cytotoxic T cells. Nature, 2005, 435: 682-686

17 Cui C, Griffiths A, Li G, Silva LM, Kramer MF, Gaasterland T, Wang XJ, Coen DM. Prediction and identification of herpes simplex virus 1-encoded microRNAs. J Virol, 2006, 80: 5499-5508

18 Griffiths-Jones S. The microRNA registry. Nucleic Acids Res, 2004, 32: D109-111

19 Griffiths-Jones S, Grocock RJ, van Dongen S, Bateman A, Enright AJ. MiRBase: microRNA sequences, targets and gene nomenclature. Nucleic Acids Res, 2006, 34: D140-144

20 Shi JD, Duan ZQ, Sun J, Wu MN, Wang B, Zhang J, Wang HX, Hu $\mathrm{NZ}, \mathrm{Hu} \mathrm{YZ}$. Identification and validation of a novel microRNA-like molecule derived from a cytoplasmic RNA virus antigenome by bioinformatics and experimental approaches. Virol J, 2014, 11: 121

21 Rouha H, Thurner C, Mandl CW. Functional microRNA generated from a cytoplasmic RNA virus. Nucleic Acids Res, 2010, 38: 8328-8337

22 Varble A, Chua MA, Perez JT, Manicassamy B, Garcia-Sastre A, tenOever BR. Engineered RNA viral synthesis of microRNAs. Proc Natl Acad Sci USA, 2010, 107: 11519-11524

23 Shapiro JS, Varble A, Pham AM, Tenoever BR. Noncanonical cytoplasmic processing of viral microRNAs. RNA, 2010, 16: 2068-2074

24 Varble A, ten Oever BR. Implications of RNA virus-produced miRNAs. RNA Biol, 2011, 8: 190-194

25 Usme-Ciro JA, Campillo-Pedroza N, Almazan F, Gallego-Gomez JC. Cytoplasmic RNA viruses as potential vehicles for the delivery of therapeutic small RNAs. Virol J, 2013, 10: 185

26 Zhang Y, Fan M, Geng G, Liu B, Huang Z, Luo H, Zhou J, Guo X, Cai W, Zhang H. A novel HIV-1-encoded microRNA enhances its viral replication by targeting the TATA box region. Retrovirology, 2014, 11: 23

27 Kaul D, Ahlawat A, Gupta SD. HIV-1 genome-encoded hiv1-mir-H1 impairs cellular responses to infection. Mol Cell Biochem, 2009, 323: 143-148

28 Kincaid RP, Burke JM, Sullivan CS. RNA virus microRNA that mimics a B-cell oncomiR. Proc Natl Acad Sci USA, 2012, 109: 3077-3082

29 Rosewick N, Momont M, Durkin K, Takeda H, Caiment F, Cleuter Y, Vernin C, Mortreux F, Wattel E, Burny A, Georges M, van den Broeke A. Deep sequencing reveals abundant noncanonical retroviral microRNAs in B-cell leukemia/lymphoma. Proc Natl Acad Sci USA,
2013, 110: 2306-2311

30 Hussain M, Torres S, Schnettler E, Funk A, Grundhoff A, Pijlman GP, Khromykh AA, Asgari S. West Nile virus encodes a microRNA-like small RNA in the $3^{\prime}$ untranslated region which up-regulates GATA4 mRNA and facilitates virus replication in mosquito cells. Nucleic Acids Res, 2012, 40: 2210-2223

31 Hussain M, Asgari S. MicroRNA-like viral small RNA from dengue virus 2 autoregulates its replication in mosquito cells. Proc Natl Acad Sci USA, 2014, 111: 2746-2751

32 Li SC, Shiau CK, Lin WC. Vir-Mir db: prediction of viral microrna candidate hairpins. Nucleic Acids Res, 2008, 36: D184-189

33 Jiang P, Wu H, Wang W, Ma W, Sun X, Lu Z. MiPred: classification of real and pseudo microRNA precursors using random forest prediction model with combined features. Nucleic Acids Res, 2007, 35: W339-344

34 Gkirtzou K, Tsamardinos I, Tsakalides P, Poirazi P. MatureBayes: a probabilistic algorithm for identifying the mature miRNA within novel precursors. PLoS One, 2010, 5: e11843

35 Bayes-SVM-MiRNA web server v1.0. http://wotan.Wistar.Upenn. Edu/bayessvmmirna find/

36 Seo GJ, Chen CJ, Sullivan CS. Merkel cell polyomavirus encodes a microRNA with the ability to autoregulate viral gene expression. Virology, 2009, 383: 183-187

37 Lewis BP, Shih IH, Jones-Rhoades MW, Bartel DP, Burge CB. Prediction of mammalian microRNA targets. Cell, 2003, 115: 787-798

38 Rehmsmeier M, Steffen P, Hochsmann M, Giegerich R. Fast and effective prediction of microRNA/target duplexes. RNA, 2004, 10: 1507-1517

39 Berezikov E, Cuppen E, Plasterk RH. Approaches to microRNA discovery. Nat Genet, 2006, 38(Suppl): S2-7

40 Singh J, Singh CP, Bhavani A, Nagaraju J. Discovering microRNAs from Bombyx mori nucleopolyhedrosis virus. Virology, 2010, 407: 120-128

41 Hussain M, Taft RJ, Asgari S. An insect virus-encoded microRNA regulates viral replication. J Virol, 2008, 82: 9164-9170

42 Besecker MI, Harden ME, Li G, Wang XJ, Griffiths A. Discovery of herpes B virus-encoded microRNAs. J Virol, 2009, 83: 3413-3416

43 Wong G, Kobinger GP, Qiu X. Characterization of host immune responses in Ebola virus infections. Expert Rev Clin Immunol, 2014, 10: 781-790

Open Access This article is distributed under the terms of the Creative Commons Attribution License which permits any use, distribution, and reproduction in any medium, provided the original author(s) and source are credited.

\section{Supporting Information}

Table S1 The target genes of EBOV-encoded miRNAs

Figure S1 Knockdown of Dicer reduces the expression of EBOV-encoded miRNAs. A, Dicer protein was knocked down by RNAi and confirmed by Western blot. GAPDH was used as a loading control. B, Dicer mRNA was knocked down by RNAi and confirmed by qRT-PCR. Significant differences in expression levels were detected by Student's $t$-test $(*, P<0.05 ; * *, P<0.01 ; * * *, P<0.001)$.

Figure S2 The EBOV-encoded miRNAs levels in HEK293T cells. A, Quantitative RT-PCR analysis of EBOV-encoded miRNAs levels in HEK293T cells treated with mimic NC, or EBOV-miRNAs mimics. B, Quantitative RT-PCR analysis of EBOV-encoded miRNAs levels in HEK293T cells treated with pcDNA6.2-GW/EmGFP, or pcDNA6.2-GW/EmGFP-EBOV-pre-miRNAs. Significant differences in expression levels were detected by Student's $t$-test (*, $P<0.05 ; * *, P<0.01 ; * * *, P<0.001)$.

The supporting information is available online at life.scichina.com and link.springer.com. The supporting materials are published as submitted, without typesetting or editing. The responsibility for scientific accuracy and content remains entirely with the authors. 